\title{
Tunable Demultiplexer Study in 2D Square Photonic Crystal
}

\author{
F.Z. Mirouh, M. Bouchemat, M.R. LebBal* And T. Bouchemat \\ Electronics Department, University Frères Mentouri of Constantine, 25000 Constantine, Algeria
}

\begin{abstract}
We propose a novel designed structure which is a demultiplexer of $2 \mathrm{D}$ square photonic crystal of circular rods embedded in air that have locally three cavities with different sizes, refractive indexes, and also appropriately sized adjacent rods. Firstly, for one constant refractive index $n(n=3.3763)$ and with individual radius for each cavity and their adjacent rods, we have three different output resonant wavelengths; it means that this structure drops the desired wavelength depending on the geometric parameters. We have optimized this structure by varying locally the refractive index for each of the resonant cavity. In this case, we have obtained in one structure i.e. a single refractive index for all the structures except the cavities of course, three different zones with specific geometric and physical properties. As a result, three wavelengths have been extracted from these cavities. The corresponding optical transmission powers and $Q$ factors are $T_{1}=83.54 \%, T_{2}=100 \%, T_{3}=81.97 \%$, and $Q_{1}=2690.3, Q_{2}=3248.8$, $Q_{3}=8212.5$, respectively. By changing both the size and refractive index of the cavity and the size of their adjacent rods, the structure is able to drop the desired wavelength, and the tunability property is achieved.
\end{abstract}

DOI: 10.12693/APhysPolA.135.613

PACS/topics: photonic bandgap, demultiplexers, waveguides, microcavity, filters

\section{Introduction}

Photonic crystals (PhC), known as periodic structures, are materials with modulation property of the refractive index which can stop propagation in some frequency's bands. Therefore, this gives rise to stop-bands for electromagnetic waves $[1,2]$. In recent years, photonic bandgap (PBG) structures have attracted much attention because they have an ability to realize an important $Q$-factor. These materials have been proposed to have many novel applications, for their ability to control light-wave propagation. The photonic crystal structures are very suitable candidates for realization of future passive and active optical devices. Several research works using the photonic crystals, have been undertaken due to their band structure and the confinement of light. Among these researches, they have proposed a new approach such as modifying L2 geometry where the nearest neighbor holes of the cavity are deformed to elliptical shape and their numbers were varied [3], by selective air-hole infiltration [4] or by modifying position and size of the holes surrounding the L3 cavity [5]. On the other hand, they have been interested on the design and optimization of the $\mathrm{PhC}$ cavity to realize higher $Q$ factor and transmission of resonant wavelengths. There are many possible device applications of compact and efficient PhCS nanocavities [6]. When we introduce the defects in the $2 \mathrm{D}$ lattice case, we can obtain various optical components which can be realized $[7,8]$. So, it is possible to fabricate sensors [9], resonant cavities, add-drop filters [10], power splitters [11, 12], channel drop filters [13, 14], multiplexers, and demultiplexers [15]. These devices are made as promising applications for photonic integrated circuits (PICs) on a semiconductor substrate.

*corresponding author; e-mail: lebbalmohamedredha@yahoo.fr
Photonic crystal microcavities have been studied widely for their ability to confine light selectively at different operating wavelengths. This property is highly desirable in the application areas of wavelength division multiplexing and filtering. Several authors have reported photonic crystal cavities with high $Q$-values and various techniques have been used to couple light into the cavities [16]. For wavelength division multiplexing (WDM) systems, we need to use basic functions such as filters, modulators, sources, photodetectors, and demultiplexers [17]. Demultiplexer as an essential element of such systems is used for selecting a channel with a specific wavelength. Demultiplexing operation may enable us to extract one frequency from one waveguide and to send it to another waveguide [18]. So far, several topologies have been proposed for demultiplexer designing, such as using line defect $\mathrm{PhC}$ waveguides $[19,20]$, coupled cavity $\mathrm{PhC}$ waveguides [21], directional coupling [22, 23], and ring resonators [24]. When the resonant cavities are coupled to the waveguides, they can be used as frequency selecting devices. In the specific resonant frequency, the resonant cavity localizes electromagnetic energy from an input waveguide into the cavity and then transmits it to the output waveguide.

In this paper, we propose a new approach to obtain high $Q$-factor and transmission response. We have to present an add-drop demultiplexer structure which has been designed by using three different radii of resonant cavities. In Sect. 1 of simulation, we have set these radii as $r_{1}=0.04 a, r_{2}=0.06 a$ and $r_{3}=0.08 a$. Moreover, we have optimized our structure by modifying the radius of the adjacent rods of the cavities. The aim of this setting of size is to tune and increase the transmission efficiency of the dropped wavelengths. So for each cavity, we have obtained one different wavelength which is detected by the monitor placed in the output port. In Sect. 2, we have varied the refractive indexes of the cavities to obtain more power transmission efficiencies and 
higher $Q$-factors. In our structure, with each of the previous cavities, we have set the adjacent rods radius and refractive indexes for $r_{1}:\left(r, n_{1}\right)=(0.26 \mu \mathrm{m}, 3.46), r_{2}$ : $\left(r, n_{2}\right)=(0.24 \mu \mathrm{m}, 3.44)$, and finally for $r_{3}:\left(r, n_{3}\right)=$ $(0.18 \mu \mathrm{m}, 3.4)$. This optimization of structure geometry is needed to obtain high transmission efficiency and $Q$-factor which is very significant one as it will be seen later. The PhC structure was analyzed by using twodimensional finite difference time domain (2D-FDTD) method $[25,26]$. The simulations were carried out with Fullwave software [27] and plane wave expansion (PWE) method [28]. To ensure no back reflection in the limit of the analyzed region, a Berenger perfectly matched layer (PML) was located around the whole structure as absorbing boundary condition [29].

\section{Design of the photonic crystal demultiplexer}

A $2 \mathrm{D}$ photonic crystal in a square lattice of dielectric rods in air with constant lattice $a=0.58 \mu \mathrm{m}$ and radius $r=0.18 a$ [30], Fig. 1a where $a$ is the lattice constant, while the refractive index $n$ is set to 3.3763 . For a 2D$\mathrm{PhC}$ with $20 \times 20$ square lattice, in Fig. 1a, we have a large photonic band gap at the normalized frequency range of 0.309-0.442 whose corresponding wavelength spans from $1.312 \mu \mathrm{m}$ to $1.877 \mu \mathrm{m}$ for TE polarization (magnetic field

(a)

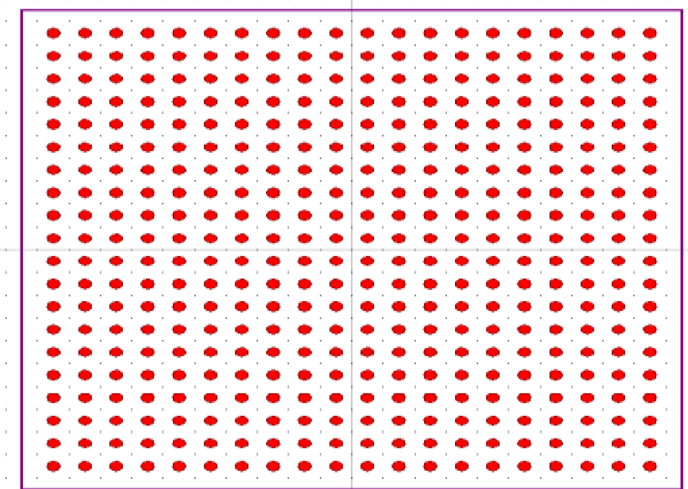

(b)

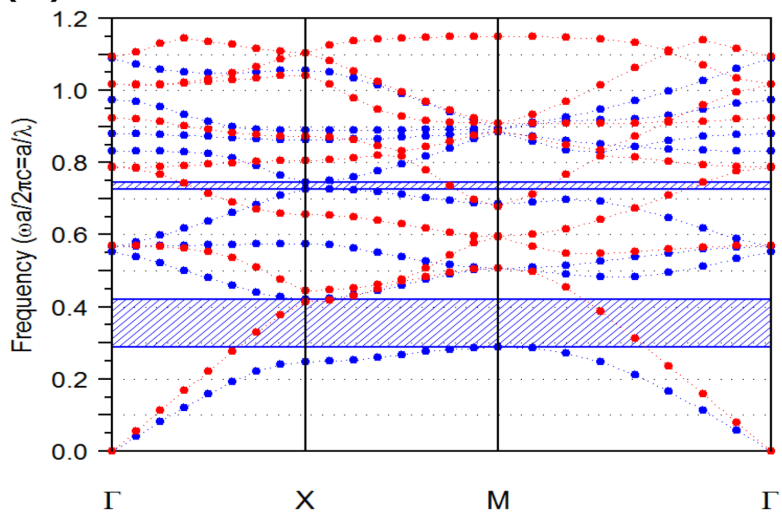

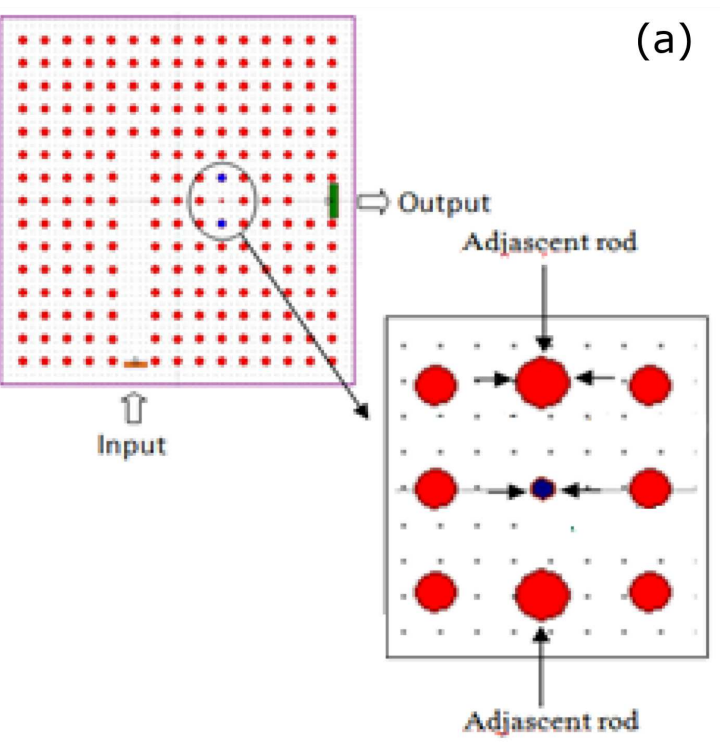

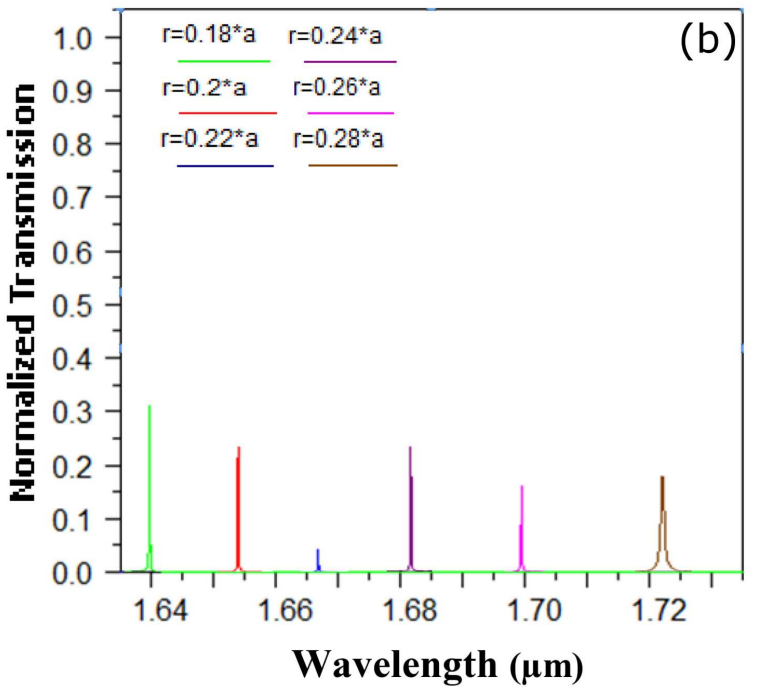

Fig. 2. (a) Typical resonator with one output port. (b) Transmission response for different adjacent rods radii for $r=0.08 a$.

parallel to the axis of dielectric rods), but no gap for TM modes with this refractive index contrast and relatively small $r / a$, where $\lambda$ is the wavelength in free space as shown in Fig. 1b.

Now, we consider a ring resonator obtained from a previous structure with $15 \times 15$ rods as shown in Fig. 2a. We have locally created a defect by changing the size of rod in the cavity, so the radius is set to $r=0.08 a$. This defect located in the centre of the resonant cavity will be coupled with an input waveguide. The in-line quasi waveguide (waveguide in the left side) is formed by removing one row of rods.

\section{Simulation results}

The aim of this study is to investigate the significant feature of the structure. The resonant wavelength can be tuned by varying the cavity's parameters. In the next (b) Band diagram of $20 \times 20$ square lattice without defects by PWE method. 
section, the effect of varying the cavity's radius and their adjacent rods on cavity resonator performance will be studied. Furthermore, later, we will describe the effect of varying the refractive index of the cavity.

\subsection{Cavity's radius and adjacent rods radius effect}

First, we vary the adjacent rods radius $r$ of the cavity. Six different curves are displayed in Fig. 2b for $r$ from $0.18 a$ to $0.28 a$. The output wavelengths which have been removed from the input waveguide to the output one through the previous cavity are shown for different radii as seen in Fig. 2b. The output wavelengths are shifted to the higher values when we increase the adjacent rods radius. To obtain the higher transmission power efficiency, adjacent rods radius has to be left in $0.18 a$ in the proposed cavity. We denote the corresponding transmission power efficiency $T=30.16 \%$ and $Q=8199$ for the resonance wavelength $\lambda=1.6399 \mu \mathrm{m}$.

In the similar way, we have created two local defects by changing the cavity's radius to $r_{1}=0.04 a$ and $r_{2}=0.06 a$ and then for obtaining power transmission and coupling efficiency to outports, we have also changed the radius
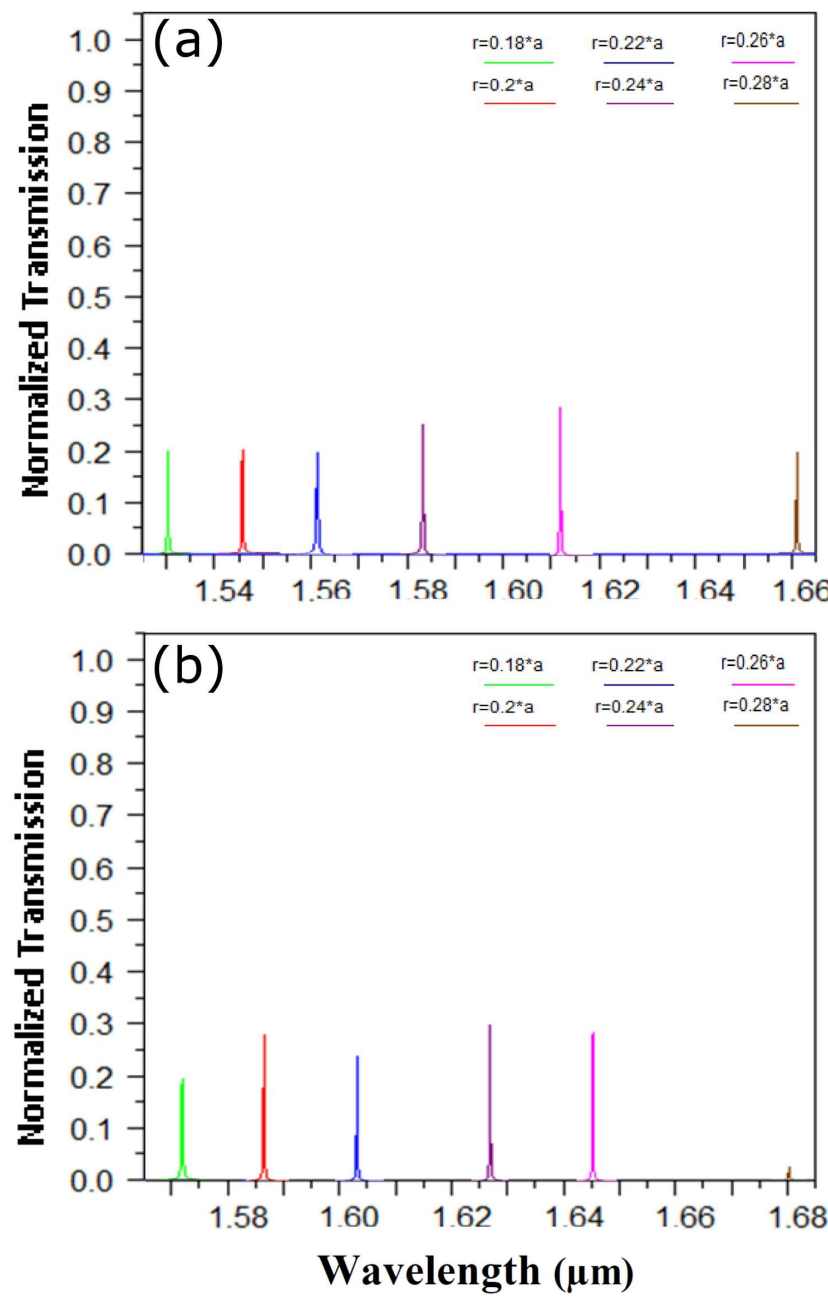

Fig. 3. Transmission response of different adjacent rods radii for: (a) $r_{1}=0.04 a$, (b) $r_{2}=0.06 a$.

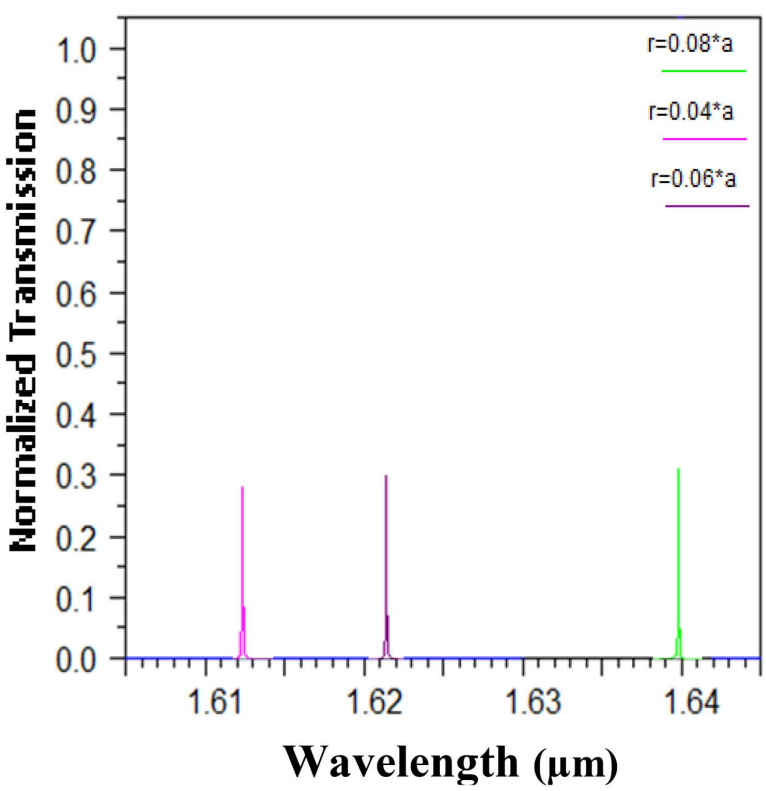

Fig. 4. Optimized optical power transmission for the three cavities sizes.

of adjacent rods for these point defects to: $r=0.26 a$ and $0.24 a$, respectively. Figure $3 \mathrm{a}$ and b shows the output wavelengths and transmission power efficiencies of the resonators with previous cavities radii, respectively. Here, the resulted wavelengths are also shifted to the higher values. The output resonant wavelengths are $\lambda_{1}\left(r_{1}\right)=1.6127 \mu \mathrm{m}, \lambda_{2}\left(r_{2}\right)=1.6212 \mu \mathrm{m}$, the power efficiencies are $T=28.81 \%$ and $29.81 \%$, and $Q_{1}=8064$, $Q_{2}=8106$, respectively. Through the cavities, the resonant wavelengths can be removed from the input waveguides to be transmitted to the output port. It means that to trap the electromagnetic energies, the ring resonators at their resonant wavelengths are coupled to these output wavelengths. In other words, the ring resonators drop light from the left waveguides and send it to the right one. Figure 4 shows the optimized optical power transmission of the structure shown in Fig. 2a for the sizes of three cavities. We denote the tunability property by increase and decrease of the cavity size, respectively, so the wavelength is shifted to the higher and lower values.

\subsection{Cavity refractive index effect}

In this section, we will show another parameter which has an effect on the resonant wavelength behaviour of the demultiplexer. By varying the refractive index of the cavity, we will investigate and display the resonance response of the structure. We can create different refractive indexes in reality by using electro-optic (E-O) or thermo-optic (T-O) material. We utilize electro-optic materials which change their refractive indexes in response to external electric field. Moreover, we can use the T-O effect caused by two-photon absorption (TPA) in Si to control the resonator's refractive index through the heat generated by optically produced carriers [31]. 


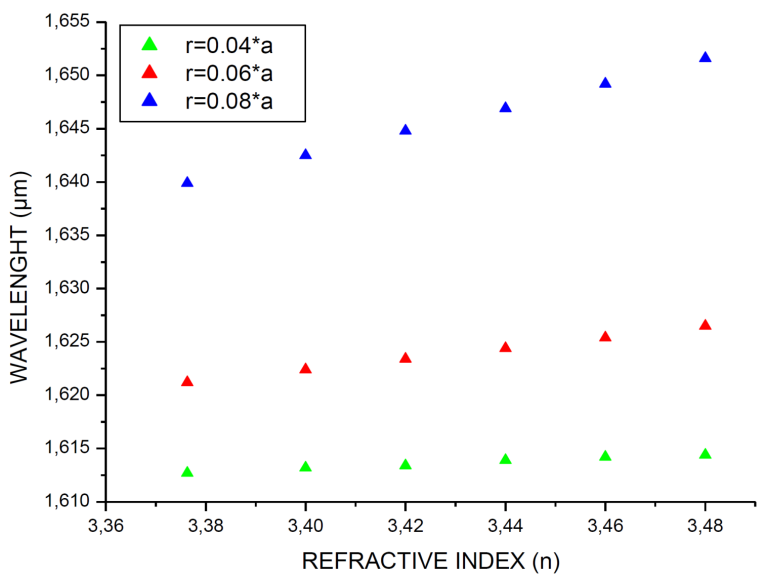

Fig. 5. Variation of resonance wavelength with the refractive index.

TABLE I

Transmission and $Q$-factor for the three resonators.

\begin{tabular}{c|c|c|c|c}
\hline \hline $\begin{array}{c}\text { Cavity } \\
\text { radius }[\mu \mathrm{m}]\end{array}$ & $\begin{array}{c}\text { Refractive } \\
\text { index } n\end{array}$ & $\lambda[\mu \mathrm{m}]$ & $\begin{array}{c}\text { Efficiency } \\
T[\%]\end{array}$ & $Q$-factor \\
\hline$r_{1}=0.04 a$ & 3.46 & 1.6142 & $99.06 \%$ & 5380.67 \\
$r_{1}=0.06 a$ & 3.44 & 1.6244 & $98.36 \%$ & 5414.66 \\
$r_{1}=0.08 a$ & 3.4 & 1.6425 & $96.13 \%$ & 5475
\end{tabular}

The previous typical ring resonator of Fig. 2a is used one more time. We will study the cavity refractive index effect from $n=3.4$ to 3.48 [28] with the different cavity radii as shown in Sect. $1\left(r_{1}=0.04 a, r_{2}=0.06 a\right.$, and $\left.r_{3}=0.08 a\right)$. By introducing variation in cavity refractive index, the resonant wavelength can be tuned. As shown in Fig. 5, the resonant wavelengths are increased and shifted to the higher values by raising refractive index accordingly. We have obtained resonance wavelengths of $\lambda_{1}=1.6142 \mu \mathrm{m}, \lambda_{2}=1.6244 \mu \mathrm{m}$ and $\lambda_{3}=1.6425 \mu \mathrm{m}$ for $r_{1}=0.04 a, r_{2}=0.06 a$, and $r_{3}=0.08 a$, while the corresponding normalized transmissions are $T_{1}=99.06 \%$, $T_{2}=98.36 \%$ and $T_{3}=96.13 \%$, respectively. In Fig. 5, it is obvious that when the refractive index of the cavity increases, the resonant wavelengths curves shift to right (greater wavelengths), which means that this structure is tunable for desired wavelengths. The optimized result cases are shown in Table I above. The higher normalized optical power transmissions response of the ring resonator of Fig. 2a with $n=3.46, n=3.44$ and $n=3.4$ are displayed in Fig. 6, in which we have three different curves for the three resonant wavelengths.

In this study we propose a design of the demultiplexer as shown in Fig. 7a. Three ring resonators of cavities radii, $r_{1}=0.04 a, r_{2}=0.06 a$, and $r_{3}=0.08 a$ with substitution of the cavities rods refractive index by the three values have been investigated in the previous section. We have used the perfect square lattice periodic PhC structure which consists of $25 \times 19$ rods in $X$ and $Y$ directions, respectively. The time step is chosen to 0.01 . Power monitors were placed at outports $B_{n}:\left(B_{1}, B_{2}\right.$, $\left.B_{3}\right)$ to collect the normalized transmitted spectral power

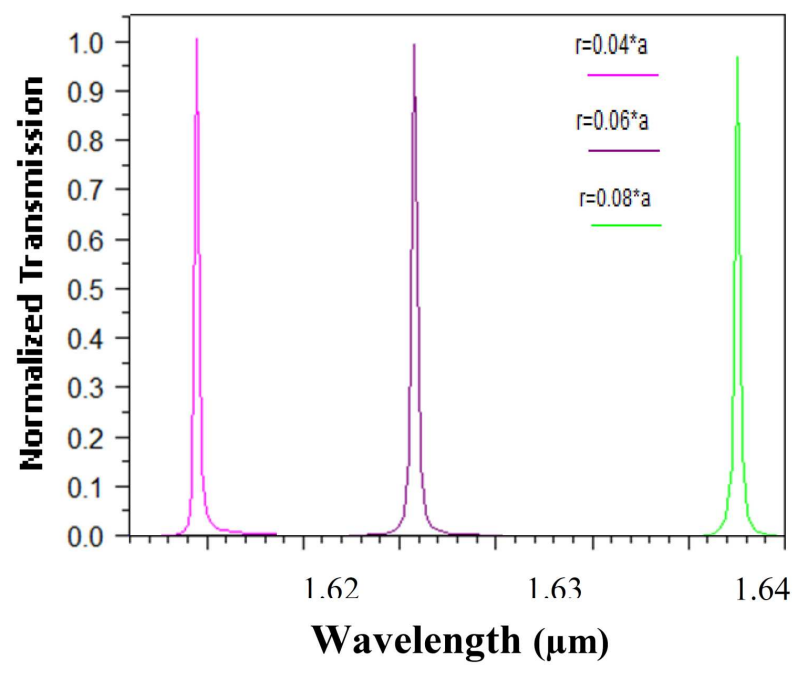

Fig. 6. Optimized optical power transmission for the three cavities refractive indexes.
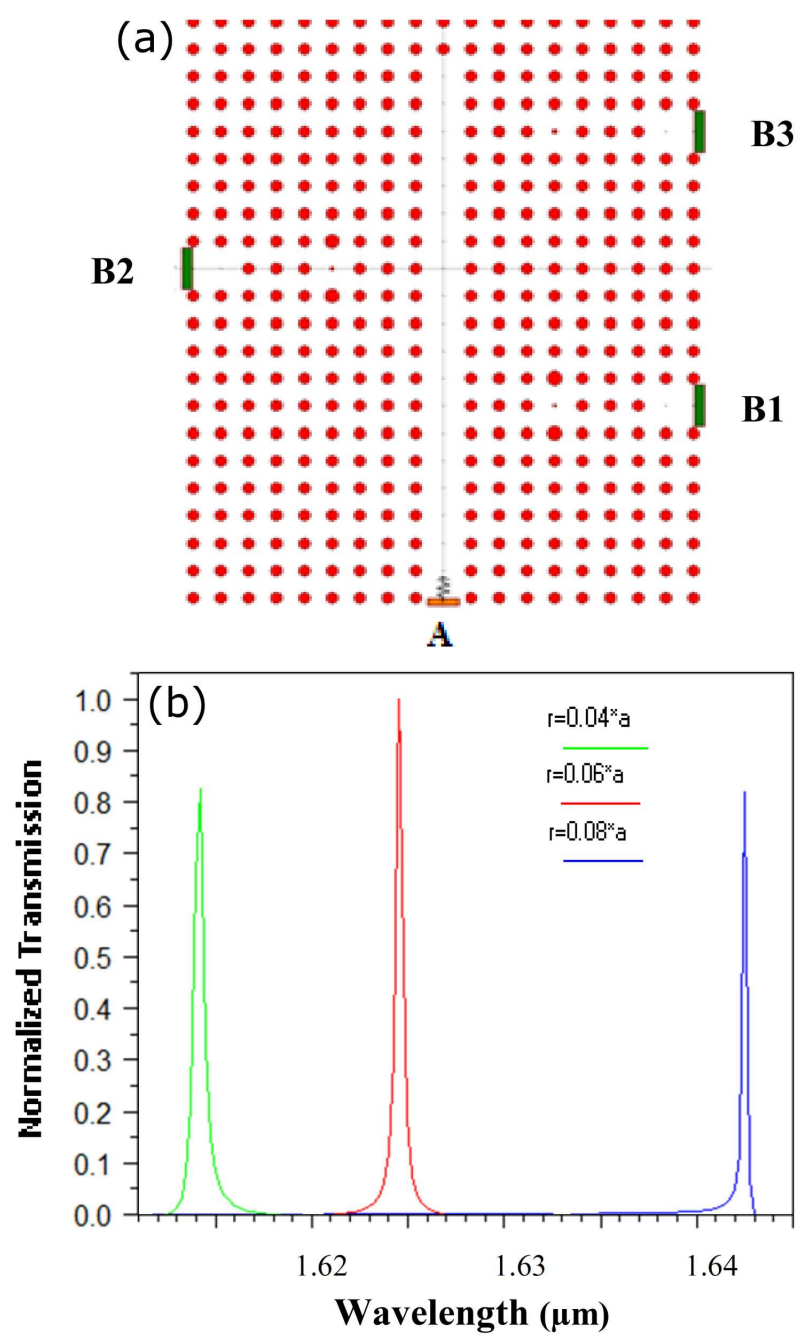

Fig. 7. (a) Schematic of the proposed structure of PhC-demultiplexer, (b) optimized optical power transmission response of the three resonators with different refractive indexes. 
TABLE II

Transmission and $Q$-factor for the optimized structure.

\begin{tabular}{c|c|c|c|c}
\hline \hline $\begin{array}{c}\text { Cavity } \\
\text { radius }[\mu \mathrm{m}]\end{array}$ & $\begin{array}{c}\text { Refractive } \\
\text { index } n\end{array}$ & $\lambda[\mu \mathrm{m}]$ & $\begin{array}{c}\text { Efficiency } \\
T[\%]\end{array}$ & $Q$-factor \\
\hline$r_{1}=0.04 a$ & 3.46 & 1.6142 & $83.54 \%$ & 2690.3 \\
$r_{2}=0.06 a$ & 3.44 & 1.6244 & $100 \%$ & 3248.8 \\
$r_{3}=0.08 a$ & 3.4 & 1.6425 & $81.97 \%$ & 8212.5
\end{tabular}

(a)

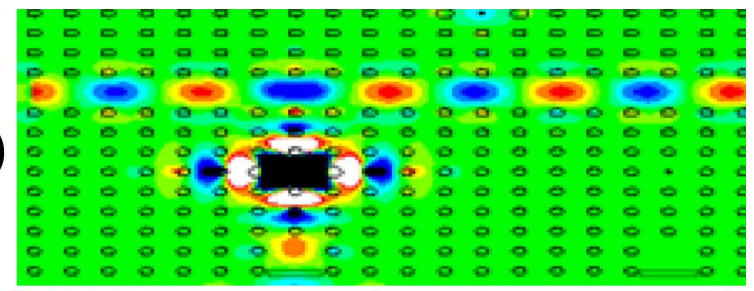

(b)

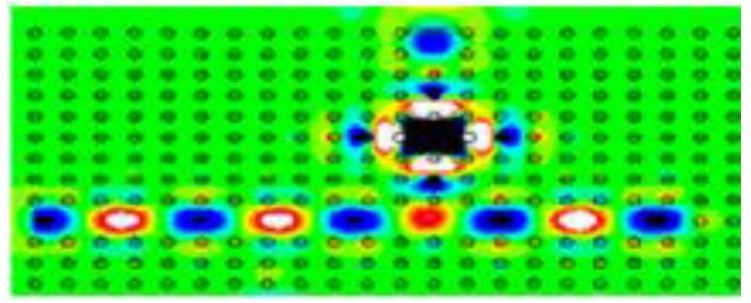

(c)

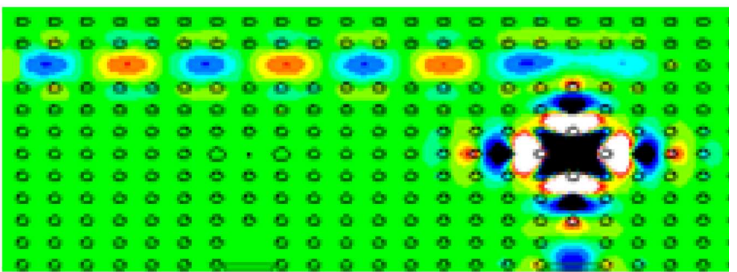

(d)

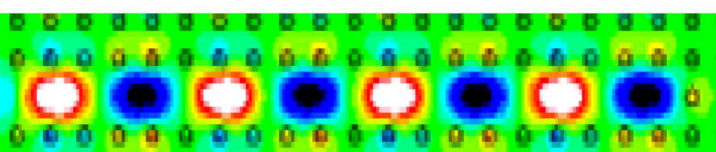

)

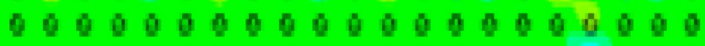

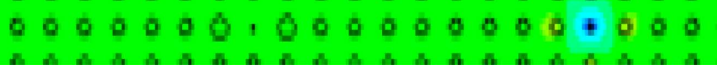

Fig. 8. Electric field distributions of the structure for on-resonant wavelengths: for: (a) $\lambda_{1}=1.6142 \mu \mathrm{m}$, (b) $\lambda_{2}=1.6244 \mu \mathrm{m}$, (c) $\lambda_{3}=1.6425 \mu \mathrm{m}$, and (d) electric field distribution of the structure for off-resonant wavelength $\lambda=1.645 \mu \mathrm{m}$.

densities. With a transverse electric (TE) polarized to the port $-A$ - we have applied the input Gaussian signal. The computational method used is based on a $2 \mathrm{D}$ FDTD method algorithm using perfectly matched layers (PML) as absorbing boundaries. $Q$-factor is calculated with $Q=\lambda / \Delta \lambda$, where $\lambda$ and $\Delta \lambda$ are central wavelength and full width at half power of output, respectively. Figure $7 \mathrm{~b}$ shows the transmission spectrum of the demultiplexer of Fig. 7a. Three different curves are displayed in Fig. $7 \mathrm{~b}$ for $n=3.46, n=3.44$, and finally $n=3.4$. As seen in Fig. 7b, the proposed structure, when simulated with the different refractive indexes can select the wavelengths $\lambda_{1}=1.6142 \mathrm{~nm}, \lambda_{2}=1.6244 \mathrm{~nm}$, and $\lambda_{3}=1.6425 \mathrm{~nm}$, respectively. The corresponding normalized optical transmission powers are $T_{1}=83.54 \%$, $T_{2}=100 \%$, and $T_{3}=81.97 \%$ accordingly. It is clear that our numerical simulations reveal the dependence of the demultiplexer's transmission spectrum on different cavity and also adjacent rods parameters, such as rods radius and cavities refractive indexes. It is shown that the resonance wavelength of our demultiplexer has been tuned by varying these different parameters appropriately. $Q$ factor values are $Q_{1}=2690.3, Q_{2}=3248.8, Q_{3}=8212.5$. In Refs. [24, 30, 32], the $Q$-factor was varied between 50 and 2814. We denote that the mean value of the transmission efficiency and $Q$-factor are $T=88.503 \%$ and $Q=4717.2$, respectively. The full structure parameters with the corresponding resonant wavelengths, $Q$-factor and the output transmission response efficiency $T$ for each cavity are displayed in Table II.

Figure $8 \mathrm{a}-\mathrm{c}$ shows the electric field distributions of the optimized proposed structure. The upper and lower waveguides and the resonators couple together to extract through ports $B_{1}, B_{2}, B_{3}$ the three on-resonant wavelengths. On the other hand, Fig. 8d displays the offresonance case for $\lambda=1.645 \mu \mathrm{m}$ in which we can deduce that there are not any coupling phenomena. As shown in Fig. 8a-c, our proposed structure is capable to directing each of the resonant wavelength to the corresponding output port $\left(B_{1}, B_{2}, B_{3}\right)$.

\section{Conclusion}

In this paper, we have optimized a novel structure of 2D photonic crystal demultiplexer in a square lattice. Three PhCs resonant cavities locally filled with three different refractive indexes instead of using different structures as shown in [30] have been studied. Our optimized structure has been used to transmit the resulted resonant wavelengths. In conclusion, we have shown that the resonant wavelength can be tuned by varying the radius and the refractive index of the cavity and their adjacent rods. The normalized optical power transmission for each output channel is optimized when we change the refractive index of the cavity. The corresponding resonant wavelengths for $r=0.04 a, 0.06 a$, and $0.08 a$ are $\lambda_{1}=1.6142 \mu \mathrm{m}, \lambda_{2}=1.6244 \mu \mathrm{m}$, and $\lambda_{3}=1.6425 \mu \mathrm{m}$, respectively. The mean value of transmission efficiency and $Q$-factor are $T=88.503 \%$ and $Q=4717.2$, respectively. We denote that this resulting $Q$-factor is higher than those reported in literature $[24,30,32]$. Such structure is promising for PICs on PhCs and other nanophotonic structures.

\section{Acknowledgments}

We would like to express our deepest appreciation to our research manager, Professor Mohamed Bouchemat, who has invested his full effort in guiding us to achieve the goal and whose suggestions and encouragement helped us especially in writing this paper. Furthermore, we would 
also like to acknowledge the crucial role of our colleague Dr. Mohamed Redha Lebbal who assisted the research and shared his wisdom during the course of this research. We are also grateful to Professor Touraya Bouchemat for her assistance to moderate this paper and improve the manuscript. We thank all those who provided us with the opportunity to complete this research.

\section{References}

[1] N.J. Florous, K. Saitoh, M. Koshiba, IEEE Photon. Technol. Lett. 17, 2316 (2005).

[2] J.D. Joannopoulos, R.D. Meade, J.N. Winn, S.G. Johnson, Photonic Crystals: Molding the Flow of Light, Princeton Univ. Press, Princeton (NJ) 1995.

[3] A. Benmerkhi, M. Bouchemat, T. Bouchemat, Photon. Nano-Fundamentals Appl. 20, 7 (2016).

[4] S.T. Hanic, C.M.D. Sterke, Sensors 13, 3262 (2013).

[5] Y. Liu, H.W.M. Salemink, Europhys. Lett. 107, 34008 (2014).

[6] A. Benmarkhi, M. Bouchemat, T. Bouchemat, Optik 125, 6223 (2014).

[7] J.D. Joannopoulos, S.G. Johnson, R.D. Meade, J.N. Winn, Photonic Crystals: Molding the Flow of Light, 2nd ed., Princeton Univ., 2008.

[8] P.R. Villeneuve, S. Fan, J.D. Joannopoulos, Phys. Rev. B 54, 7837 (1996).

[9] T. Zouache, A. Hocini, A. Harhouz, R. Mokhtari, Acta Phys. Pol. A 131, 68 (2017).

[10] C. Manolatou, M.J. Khan, S. Fan, P.R. Villeneuve, H.A. Haus, J.D. Joannopoulos, IEEE J. Quant. Electron. 35, 1322 (1999).

[11] A. Ghaffari, F. Monifi, M. Djavid, M.S. Abrishamian, J. Appl. Sci. 8, 1416 (2008).

[12] N. Nozhat, N. Granpayeh, J. Appl. Sci. 7, 3576 (2007).

[13] C.C. Wang, L.W. Chen, Physica B 405, 1210 (2010).

[14] M. Djavid, A. Ghaffari, F. Monifi, M.S. Abrishamian, Physica E 40, 3151 (2008).
[15] G. Manzacca, D. Paciotti, A. Marchese, M.S. Moreolo, G. Cincotti, Photon. Nano-Fundament. Appl. 5, 164 (2007).

[16] R. Moukhtari, A. Hocini, D. Khedrouche, Acta Phys. Pol. A 129, 556 (2016).

[17] P. Pottier, C. Seassal, X. Letartre, J.L. Leclercq, P. Viktorovitch, D. Cassagne, C. Jouanin, J. Lightw. Technol. 17, 2058 (1999).

[18] Y. Pennec, J.O. Vasseur, B. Djafari-Rouhani, L. Dobrzynski, P.A. Deymier, Sci. Rep. 65, 229 (2010).

[19] A.E. Akosman, M. Mutlu, H. Kurt, E. Ozbay, Opt. Expr. 19, 24129 (2011).

[20] X. Zhang, Q. Liao, T. Yu, N. Liu, Y. Huang, Opt. Commun. 285, 274 (2012).

[21] A. Rostami, H. Alipour Banaei, F. Nazari, A. Bahrami, Optik 122, 1481 (2011).

[22] R. Selim, D. Pinto, S.S.A. Obayya, Opt. Quant. Electron. 42, 425 (2011).

[23] M.S. Moreolo, F. Silvestri, M. Armellino, K. Hingerl, G. Cincotti, Photon. Nano-Fundament. Appl. 4, 155 (2006).

[24] M. Djavid, F. Monifi, A. Ghaffari, M.S. Abrishamian, Opt. Commun. 281, 4028 (2008).

[25] A. Taflove, S.C. Hagness, Computational Electrodynamics: The Finite-Difference Time-Domain Method, 3rd ed., Artech House, Norwood 2005.

[26] K.S. Kunz, R.J. Luebbers, The Finite-Difference Time-Domain Method for Electromagnetic, 1st ed., CRC Press, Boca Raton 1993.

[27] Fullwave, RSoft, ver. 6.1, license 16847214.

[28] J.B. Pendry, J. Phys. Condens. Matter 8, 1085 (1996).

[29] J.P. Berenger, J. Comput. Phys. 114, 185 (1994).

[30] M.R. Rakhshani, M.A. Mansouri-Birjandi, Z. Rashki, Int. Res. J. Appl. Basic Sci. 4, 976 (2013).

[31] M.R. Rakhshani, M.A. Mansouri-Birjandi, Optik 124, 5377 (2013)

[32] S. Bouamami, R. Naoum, Optik 124, 2373 (2013). 